Artigo Original

Original Article

Kátia de Freitas Alvarenga ${ }^{1}$

Eliene Silva Araújoº

Tatiana Mendes de $\mathrm{Melo}^{3}$

Maria Angelina Nardi Martinez ${ }^{4}$

Maria Cecília Bevilacqua ${ }^{5}$

Descritores

Monitoramento

Perda auditiva

Triagem neonatal

Questionários

Programa saúde da família

Audiologia

Keywords

Monitoring

Hearing loss

Neonatal screening

Questionnaires

Family health program

Audiology

Endereço para correspondência:

Kátia de Freitas Alvarenga

Departamento de Fonoaudiologia

Al. Octávio Pinheiro Brisola, 9/75, Bauru

(SP), Brasil, CEP: 17012-901.

E-mail:katialv@fob.usp.br

Recebido em: 26/8/2011

Aceito em: 9/4/2012

\section{Questionário para monitoramento do desenvolvimento auditivo e de linguagem no primeiro ano de vida}

\author{
Questionnaire for monitoring auditory and language \\ development in the first year
}

\section{RESUMO}

Objetivo: Validar um questionário de monitoramento do desenvolvimento da função auditiva e de linguagem, aplicado por agentes comunitários de saúde no primeiro ano de vida. Métodos: Setenta e seis agentes comunitários de saúde, capacitados previamente em saúde auditiva infantil, aplicaram o questionário às famílias de 304 crianças de 0 a 1 ano de idade. O questionário constitui-se de questões sobre o desenvolvimento da função auditiva e da linguagem, sendo que para todas as faixas etárias possui a questão "Seu filho ouve bem?". A validade do questionário foi averiguada por meio da análise das taxas de falso-positivo e falso-negativo das crianças investigadas por ele. Para tanto, foi utilizado um estudo duplo-cego em que todas as crianças investigadas pelo questionário foram submetidas à avaliação audiológica realizada por fonoaudiólogos. Resultados: Foram diagnosticadas quatro crianças $(1,32 \%)$ com perda auditiva sensorioneural, sendo duas unilaterais e 69 crianças $(22,7 \%)$ com perda auditiva condutiva. O questionário de acompanhamento demonstrou especificidade de $96 \%$ e sensibilidade de $67 \%$, com taxa de falso-negativo de $33 \%$, por não identificar as perdas auditivas unilaterais, e falso-positivo de 4\%. Conclusão: A utilização do questionário proposto mostrou-se viável e pertinente às ações desenvolvidas pelos agentes comunitários de saúde da Estratégia de Saúde da Família, demonstrando alta especificidade e moderada sensibilidade. A utilização do instrumento validado deve ser considerada como forma complementar aos Programas de Triagem Auditiva Neonatal, a fim de identificar as alterações auditivas de caráter adquirido ou de início tardio.

\begin{abstract}
Purpose: To validate a monitoring questionnaire about hearing and language development applied by community health agents in the first year of life. Methods: Seventy six community health agents, previously trained on infant hearing health, administered a questionnaire to the families of 304 children with ages from 0 to 1 year. The questionnaire contains questions regarding hearing and language development and, for all age groups, the question "Does your child hear well?" was presented. The validity of the questionnaire was assessed by analyzing false positive and false negative rates of the identified children. A double-blind study was conducted so that all children assessed by the questionnaire were submitted to hearing evaluation performed by audiologists. Results: Four children (1.32\%) were diagnosed with sensorineural hearing loss (two unilateral), and $69(22.7 \%)$ with conductive hearing loss. The monitoring questionnaire showed specificity of $96 \%$ and sensitivity of $67 \%$, with a false-negative rate of $33 \%$ for not identifying the unilateral hearing loss, and a false-positive rate of $4 \%$. Conclusion: The questionnaire used has shown to be feasible and relevant to actions of the community health agents of the Family Health Strategy program, with high specificity and moderate sensitivity. The use of the validated instrument should be considered to complement Newborn Hearing Screening Programs, in order to identify late onset or acquired hearing loss.
\end{abstract}

Trabalho realizado no Departamento de Fonoaudiologia, Faculdade de Odontologia de Bauru, Universidade de São Paulo - USP - Bauru (SP), Brasil; Associação de Pais e Amigos de Deficientes Auditivos de Sorocaba - APADAS - Sorocaba (SP), Brasil.

(1) Departamento de Fonoaudiologia, Faculdade de Odontologia de Bauru, Universidade de São Paulo - USP - Bauru (SP), Brasil; Centro de Pesquisas Audiológicas, Hospital de Reabilitação de Anomalias Craniofaciais, Universidade de São Paulo - USP - Bauru (SP), Brasil.

(2) Programa de Pós-Graduação (Mestrado) em Fonoaudiologia, Faculdade de Odontologia de Bauru, Universidade de São Paulo - USP - Bauru (SP), Brasil.

(3) Curso de Fonoaudiologia, Universidade de Guarulhos - UnG - Guarulhos (SP), Brasil.

(4) Departamento de Clínica Fonoaudiológica, Curso de Fonoaudiologia, Pontifícia Universidade Católica de São Paulo - PUC-SP - São Paulo (SP), Brasil; Associação de Pais e Amigos de Deficientes Auditivos de Sorocaba - APADAS - Sorocaba (SP), Brasil.

(5) Departamento de Fonoaudiologia, Faculdade de Odontologia de Bauru, Universidade de São Paulo - USP - Bauru (SP), Brasil; Programa de Pós-Graduação em Fonoaudiologia, Pontifícia Universidade Católica de São Paulo - PUC-SP - São Paulo (SP), Brasil.

Conflitos de interesses: Não 


\section{INTRODUÇÃO}

Na literatura da área, existe uma ampla discussão sobre as evidências científicas a respeito da efetividade dos programas de Triagem Auditiva Neonatal (TAN) em promoverem o diagnóstico da deficiência auditiva e possibilitarem a intervenção nos primeiros meses de vida, principalmente nos países em desenvolvimento.

O principal ponto discutido é o fato de que muitos programas não estão inseridos em programas de saúde auditiva neonatal que garantam o acesso rápido e eficaz ao diagnóstico e à (re)habilitação. Além disto, ressalta-se a dificuldade no monitoramento das crianças que passam na TAN e apresentam risco para perdas auditivas de manifestação tardia ${ }^{(1)}$.

Outros problemas são inerentes a este tipo de programa, como um percentual de recém-nascidos que não realizam a triagem auditiva e a elevada taxa de evasão dos neonatos que não dão continuidade às outras etapas do processo de identificação (reteste da TAN) e/ou diagnóstico audiológico, quando necessário. O número de desistências ao longo do processo de identificação, ou seja, crianças que não realizam o diagnóstico, é uma das dificuldades na implementação de Programas de Triagem Auditiva Neonatal tanto em nível nacional ${ }^{(2-4)}$ quanto internacional ${ }^{(5-7)}$. Neste sentido, a avaliação audiológica com fins de diagnóstico deve ocorrer poucas semanas após a saída do hospital, para minimizar o número de crianças que não retornam, pois a probabilidade de comparecer ao agendamento é maior nas primeiras semanas de $\mathrm{vida}^{(8)}$.

Ao constatar que o processo de diagnóstico dos recém-nascidos identificados na TAN já impõe dificuldades, o monitoramento daqueles que apresentaram resultado "passa" na TAN, mas que apresentam algum indicador de risco requer estratégias diferentes e eficazes. O acompanhamento periódico nos Centros de Referência de todos os recém-nascidos com indicadores de risco, mas com resultado "passa" na TAN, mostrou-se muitas vezes, uma prática inviável, criando um fluxo de agendamento que requer uma ampla equipe de profissionais especializados, tornando a relação custo-efetividade do programa muito elevada. Diante desta realidade, recomendou-se que o acompanhamento dos recém-nascidos ocorra de acordo com os indicadores de risco, ou seja, o número de avaliações e o momento em que as mesmas deverão ocorrer devem ser definidos individualmente, de acordo com as evidências científicas da possibilidade de perda auditiva com início tardio ${ }^{(9)}$. Pode-se citar como exemplos, a infecção congênita por Citomegalovírus, muitas vezes assintomática, a mutação mitocondrial A1555G e o alargamento do aqueduto vestibular (Síndrome do Aqueduto Alargado), descritos como responsáveis por $60 \%$ das perdas auditivas de aparecimento tardio ${ }^{(10,11)}$.

A experiência mostra que programas isolados de TAN estão fadados ao fracasso se não fizerem parte de um programa mais amplo de saúde auditiva que interligue os vários níveis de atenção à saúde, criando assim uma rede de identificação, orientação e apoio às famílias, diagnóstico e intervenção. O sucesso dos Programas de Saúde Auditiva depende da interrelação de vários fatores, entre eles, o apoio e a política pública para pesquisa e monitoramento, educação continuada dos profissionais envolvidos em todos os níveis de atenção à saúde e a existência de centros de excelência para diagnóstico e intervenção ${ }^{(12)}$.

Considerando a problemática exposta faz-se necessária a criação de estratégias que ampliem a identificação e a detecção precoce da deficiência auditiva em todos os níveis de atenção à saúde, e não apenas a TAN.

No Brasil, a Estratégia da Saúde da Família (ESF) surgiu em 1994, tendo como precursor o Programa de Agentes Comunitários de Saúde (PACS) implantado em 1991, apoiado em experiências internacionais e nacionais de extensão da cobertura à saúde por meio de assistência às famílias no próprio domicílio, em articulação com as unidades de saúde ${ }^{(13)}$. Neste contexto, os Agentes Comunitários de Saúde (ACS) são os profissionais da saúde que têm maior contato com a comunidade, podendo então desempenhar importante papel na saúde auditiva infantil possibilitando o diagnóstico e a intervenção na deficiência auditiva no período crítico de desenvolvimento da criança $a^{(2,14,15)}$.

A Organização Mundial da Saúde (OMS), desde 1998, recomendou o treinamento de ACS para atenção primária à audição e às afecções do ouvido, amparado por um sistema de referência e contra-referência ${ }^{(16)}$. As recomendações do International Workshop on Primary Ear and Hearing Care resultaram em três manuais publicados pela OMS que correspondem a três níveis de treinamento de agentes comunitários de saúde: básico, intermediário e avançado ${ }^{(17)}$. Nos manuais de treinamento dos níveis intermediário e avançado é proposto um questionário para identificação de alterações auditivas pelo ACS. A pergunta: "Seu filho ouve bem?" é sugerida para que a suspeita dos pais seja valorizada e verificada ${ }^{(17)}$.

A utilização de questionários aplicados aos pais é recomendada pela Academia Americana de Pediatria para os programas de identificação de alterações de desenvolvimento, com posterior encaminhamento para avaliações especializadas, somando-se à observação clínica do pediatra ${ }^{(18)}$.

Diante do exposto, o presente estudo objetivou validar um questionário de monitoramento do desenvolvimento da função auditiva e de linguagem, aplicado por Agentes Comunitários de Saúde no primeiro ano de vida.

\section{MÉTODOS}

O estudo obteve aprovação do Comitê de Ética em Pesquisa da Faculdade de Odontologia de Bauru, Universidade de São Paulo (USP-Bauru), sob número 73/2006. Os pais/responsáveis pelos participantes, assim como os agentes comunitários de saúde (ACS), consentiram com a realização da pesquisa mediante assinatura do termo de consentimento livre e esclarecido.

\section{Casuística}

Participaram deste estudo 76 ACS capacitados previamente na área de saúde auditiva infantil. O questionário foi aplicado pelos ACS às famílias de 418 crianças durante as visitas domiciliares da Estratégia de Saúde da Família. Deste total, 304 crianças (73\%) compareceram ao Núcleo de Saúde 


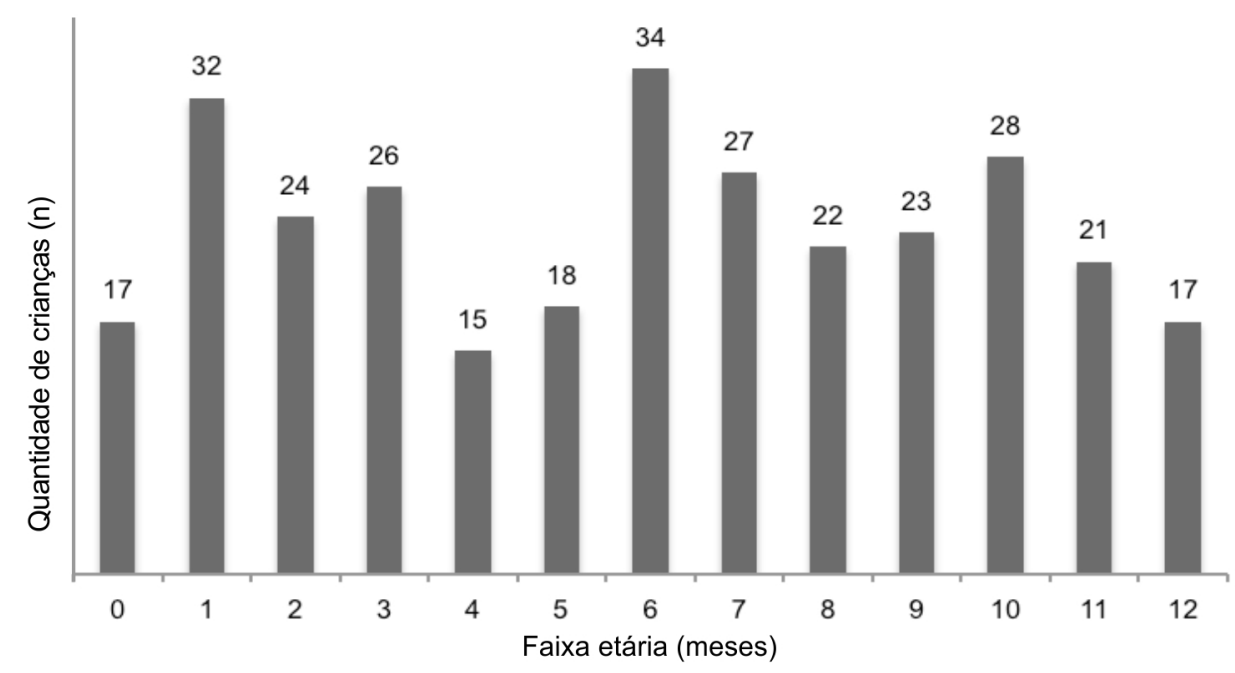

Figura 1. Distribuição das crianças participantes do estudo, de acordo com a faixa etária (meses)

e participaram da etapa de validação do questionário. A distribuição das crianças, de acordo com a faixa etária (meses), está apresentada na Figura 1.

\section{Metodologia}

Os ACS que participaram do estudo haviam realizado o curso de presencial de capacitação na área de saúde auditiva infantil oferecido previamente, com carga horária de oito horas $^{(14)}$. O conteúdo ministrado foi adaptado da World Health Organization: primary ear and hearing care training resour$c e^{(17)}$, envolvendo os temas: audição e deficiência auditiva, tipos e causas de perdas auditivas, técnicas de identificação, diagnóstico e reabilitação da deficiência auditiva. Outro aspecto abordado foi o padrão de normalidade para o desenvolvimento motor e de linguagem. Os ACS receberam uma apostila com o conteúdo ministrado, para que pudessem acompanhar as atividades realizadas de forma interativa.

O questionário aplicado pelos ACS continha questões sobre o desenvolvimento da audição e linguagem, considerando os comportamentos esperados a cada mês, no período de 0 a 1 ano. A pergunta: "Seu filho ouve bem?" foi incluída em todos os meses para verificar a opinião dos pais sobre a audição de seus filhos (Anexo 1).

A validade do questionário aplicado pelos ACS como identificador de crianças com alteração auditiva foi averiguada por meio da análise das taxas de falso-positivo e falso-negativo das crianças investigadas por ele. Para tanto, foi utilizado um estudo duplo-cego em que todas as crianças investigadas pelo questionário foram submetidas, na mesma semana, à avaliação audiológica realizada por fonoaudiólogos, considerada assim, o padrão-ouro.

A avaliação audiológica foi realizada no Núcleo de Saúde no qual a Estratégia de Saúde da Família está inserida, por meio de anamnese, avaliação do comportamento auditivo com os Sons do Ling ${ }^{(19)}$, emissões otoacústicas evocadas transientes e medida da imitância acústica (timpanometria e pesquisa do reflexo acústico). Os critérios adotados para análise foram, respectivamente: comportamento auditivo de acordo com o padrão esperado para a idade, baseado em estudo nacional ${ }^{(20)}$; presença de emissões otoacústicas evocadas transientes em pelo menos três frequências não consecutivas, incluindo 4 $\mathrm{kHz}$, com reprodutibilidade $\geq 70 \%$ e relação sinal ruído $\geq 6 \mathrm{~dB}$; curva timpanométrica com complacência de 0,3 a $1,3 \mathrm{ml}$ e pressão de +90 a -100 daPa, presença de reflexos acústicos em intensidade $<100 \mathrm{~dB}$.

As crianças com alteração em qualquer um dos testes aplicados foram encaminhadas para a Clínica do Curso de Fonoaudiologia da Faculdade de Odontologia de Bauru, Universidade de São Paulo para avaliação médica e complementação da avaliação audiológica com a pesquisa dos potenciais evocados auditivos de tronco encefálico (PEATE) e audiometria com reforço visual, e caso necessário, a realização do tratamento específico. Para análise do PEATE considerou-se a presença das ondas I, III e V, com latências absolutas e intervalos interpicos dentro dos padrões de normalidade para a faixa etária e na audiometria com reforço visual, limiar de 20 dBNA nas frequências de $500 \mathrm{~Hz}$ a $4 \mathrm{kHz}$. Os fonoaudiólogos que realizaram a avaliação audiológica não tiveram contato prévio com as informações do questionário aplicado pelo ACS.

Foi considerada identificada como "de risco para alteração auditiva" a criança com pelo menos uma resposta "não" às perguntas do questionário indicadas para a idade da criança. Por outro lado, quando todas as respostas foram adequadas, a criança foi considerada como apresentando desenvolvimento esperado para a idade. Para a análise dos dados os possíveis resultados da avaliação audiológica foram: audição normal, perda auditiva condutiva, perda auditiva sensorioneural.

\section{Análise dos dados}

Os dados foram tabulados em banco de dados do Microsoft Office Excel, versão 14.0 e posteriormente submetidos à análise estatística descritiva, por meio do cálculo das taxas de sensibilidade, especificidade, falso positivo, falso negativo, valores preditivos positivos e negativos e o intervalo de confiança foi de 95\% para todas as medidas. Além disso, foi calculada a razão de verossimilhança positiva. 


\section{RESULTADOS}

$\mathrm{Na}$ avaliação audiológica, do total de 304 crianças, 75 $(24,67 \%)$ apresentaram alteração auditiva e $229(75,33 \%)$ apresentaram resultado normal. A classificação da alteração auditiva quanto ao tipo, de acordo com a avaliação audiológica está descrita na Tabela 1. No que se refere ao grau, as alterações auditivas observadas foram leve ou moderada, independentemente do tipo.

Tabela 1. Distribuição quanto ao tipo de alteração auditiva das crianças diagnosticadas

\begin{tabular}{lcc}
\hline Alteração auditiva & Número de crianças & $\%$ \\
\hline Condutiva unilateral & 28 & 37,3 \\
Condutiva bilateral & 41 & 54,7 \\
Sensorioneural unilateral & 2 & 2,7 \\
Sensorioneural bilateral & 4 & 5,3 \\
\hline Total & 75 & 100 \\
\hline
\end{tabular}

Das 69 crianças com perda auditiva condutiva devido a otite média serosa, de acordo com a avaliação audiológica e o diagnóstico realizado pelo médico otorrinolaringologista da instituição, apenas uma $(1,45 \%)$ foi identificada pelo questionário aplicado.

Considerando apenas as perdas auditivas sensorioneurais, a Tabela 2 apresenta a distribuição da casuística de acordo com os resultados obtidos no questionário de acompanhamento e na avaliação audiológica.

Tabela 2. Distribuição da casuística de acordo com os resultados obtidos no questionário de monitoramento e na avaliação audiológica, considerando as perdas auditivas sensorioneurais

\begin{tabular}{lccc}
\hline \multirow{2}{*}{ Resultado do questionário } & \multicolumn{3}{c}{ Avaliação audiológica } \\
\cline { 2 - 4 } & Normal & Alterado & Total \\
\hline Identificada como de risco & 11 & 4 & 15 \\
Identificado como sem risco & 287 & 2 & 289 \\
\hline Total & 299 & 6 & 304 \\
\hline
\end{tabular}

A análise da validade do questionário de acompanhamento aplicado pelos ACS para identificação de perda auditiva sensorioneural, incluindo o intervalo de confiança de $95 \%$ e a análise da razão de verossimilhança positiva, encontram-se na Tabela 3 .

Tabela 3. Análise da validade do questionário de monitoramento aplicado pelos Agentes Comunitários de Saúde para identificação de perda auditiva sensorioneural

\begin{tabular}{lccc}
\hline \multirow{2}{*}{ Medidas } & \multicolumn{2}{c}{$\begin{array}{c}\text { Intervalo de } \\
\text { confiança de } 95 \%\end{array}$} \\
\cline { 3 - 4 } & Taxas & $\begin{array}{c}\text { Limite } \\
\text { inferior }\end{array}$ & $\begin{array}{c}\text { Limite } \\
\text { superior }\end{array}$ \\
\hline Sensibilidade & $67 \%$ & $29 \%$ & $100 \%$ \\
Especificidade & $96 \%$ & $94 \%$ & $98 \%$ \\
Falso positivo & $4 \%$ & $1,5 \%$ & $5,8 \%$ \\
Falso negativo & $33 \%$ & $-4 \%$ & $4 \%$ \\
Valor preditivo positivo & $27 \%$ & $4 \%$ & $49 \%$ \\
Valor preditivo negativo & $99 \%$ & $98 \%$ & $100 \%$ \\
Razão de verossimilhança positiva & 18 & 8 & 41 \\
\hline
\end{tabular}

\section{DISCUSSÃO}

A utilização de questionários como procedimento de identificação de alterações no desenvolvimento auditivo em diferentes faixas etárias é amplamente discutida na literatura ${ }^{(21-24)}$.

No presente estudo foram diagnosticadas 69 crianças $(22,7 \%)$ com perda auditiva condutiva e quatro com perdas sensorioneurais $(1,32 \%)$, ambas de graus leve ou moderado.

Constatou-se uma elevada taxa de alterações de orelha média não identificadas pelo questionário (Tabela 1). Neste caso, a perda auditiva é do tipo condutiva, geralmente de grau leve, o que dificulta a identificação da mesma pelo questionário aplicado aos pais, uma vez que a criança reage ao som de intensidade média/forte, assim como observa-se a aquisição e o desenvolvimento da linguagem oral, o que torna a suspeita de alteração auditiva por parte da família extremamente difícil. Estudos anteriores demonstraram que questionários não são considerados instrumentos efetivos para a identificação de perdas auditivas do tipo condutiva, pois não diferenciam crianças com ou sem desordens condutivas ${ }^{(25,26)}$.

Contudo, o fato do questionário não identificar perdas auditivas condutivas mostrou-se uma vantagem para o programa que utilizá-lo, uma vez que a identificação precoce da otite média serosa em crianças assintomáticas não melhora os resultados da intervenção; sendo assim, a triagem auditiva nesta situação não é recomendada ${ }^{(27)}$.

De maneira geral, os estudos apontaram que questionários voltados à identificação de alteração na função auditiva apresentam especificidade entre 94 a $100 \%$ e sensibilidade variando de 10 a $75 \%{ }^{(21-24)}$. Esta variabilidade na sensibilidade pode ser justificada pelas diferenças metodológicas com relação à faixa etária estudada, compreendendo entre seis meses e sete anos de idade; ou com relação ao valor de audibilidade considerado como perda de audição, que em alguns estudos foram considerados envolveu limiares acima de $40 \mathrm{dBNA}$ segundo a definição de perda incapacitante da $\mathrm{OMS}^{(16)}$ ou acima de $20 \mathrm{dBNA}$ quando consideradas crianças de zero a um ano ${ }^{(28)}$.

Conforme observado nas Tabelas 2 e 3 , quando considerada a perda auditiva sensorioneural, o questionário aplicado demonstrou especificidade de $96 \%$ e sensibilidade de $67 \%$, condizente com o descrito em estudos anteriores que usaram o questionário como metodologia ${ }^{(21-24)}$. É importante ressaltar que a sensibilidade do instrumento, bem como a taxa de falso negativo obtida (33\%), foram resultado da não identificação das duas crianças com perda auditiva sensorioneural unilateral. Isso não deve ser analisado como uma fragilidade no procedimento utilizado, pois um ponto que deve ser considerado é a percepção da família sobre o desenvolvimento da função auditiva e da linguagem da criança ${ }^{(23)}$.

De modo geral, independentemente do nível sócio-econômico, a não reação ao som ${ }^{(29)} \mathrm{e}$ a não aquisição da linguagem ${ }^{(30)}$ são os principais indícios que levam as famílias a suspeitarem de alterações auditivas. Na presença de perda auditiva unilateral tais indícios são minimizados, o que dificulta a suspeita por parte da família. A aplicação mensal do questionário aumenta a possibilidade de identificação destas alterações sem aumentar o custo do procedimento já que a proposta é que tal ação seja incluída na rotina dos ACS. 
No que se refere à taxa de falso positivo (4\%), esta se encontra dentro do proposto pelos Comitês para os programas de triagem auditiva, sugerindo, assim, que o procedimento não implica em custos excessivos em virtude de uma grande quantidade de crianças encaminhadas para a avaliação audiológica e que não apresentam alteração. Tal dado é evidenciado também pela razão de verossimilhança positiva, definida como a chance do resultado positivo vir a diagnosticar a verdadeira doença e não um resultado falso. $\mathrm{O}$ valor obtido foi 18 , o que equivale dizer que a chance do resultado no questionário ser um verdadeiro-positivo é 18 vezes maior do que a chance de representar um resultado falso-positivo.

Considerando os resultados expostos, podemos considerar efetiva a utilização do questionário, não como uma forma de triagem auditiva, mas sim de maneira complementar aos Programas de TAN, utilizando o instrumento como forma de monitorar o desenvolvimento da função auditiva e de linguagem de todas as crianças no primeiro ano de vida, independentemente de terem sido submetidas ou não à TAN, por profissionais não especializados, como os ACS. A característica de funcionamento da ESF permite o acompanhamento mensal por parte dos ACS, com a aplicação do questionário nas visitas domiciliares. O questionário assume, assim, um papel também informativo para a família, que começa a valorizar a audição e observar as respostas das crianças. O questionário teve, portanto, uma função educadora e multiplicadora na prevenção da deficiência auditiva.

No entanto, é necessário ter clareza que ao utilizar este instrumento é possível identificar perdas auditivas bilaterais de maior severidade, não detectando assim casos de perdas leves ou perdas auditivas unilaterais. No primeiro ano de vida a suspeita da família de alterações auditivas leves é extremamente incomum devido ao fato que o desenvolvimento da fala e linguagem nestes casos podem ser semelhantes ao de crianças com audição normal ${ }^{(22)}$.

A proposta de restringir o monitoramento para o primeiro ano de vida tem como objetivo a inserção gradativa deste procedimento na rotina dos ACS, visando a melhor organização e conhecimento das dificuldades. Entretanto, é fato que no futuro esta faixa etária deverá ser ampliada, tendo em vista a ocorrência de perdas auditivas adquiridas ou de manifestação tardia.

\section{CONCLUSÃO}

A utilização do questionário proposto neste estudo mostrou-se viável e pertinente às ações desenvolvidas pelos Agentes Comunitários de Saúde das equipes da Estratégia de Saúde da Família. Associado a isto, despertou nas famílias o interesse e a observação do desenvolvimento da audição de seus filhos, além de questionamentos sobre as causas da perda auditiva, desempenhando, assim, uma função educadora e multiplicadora na prevenção da deficiência auditiva.

\section{AGRADECIMENTOS}

Agradecemos o Conselho Nacional de Desenvolvimento Científico e Tecnológico (CNPq), pelo financiamento, processo número 403719/2004-6.
* KFA contribuiu substancialmente para aquisição, análise e interpretação dos dados, redação do artigo, revisão crítica e aprovação da versão final do manuscrito. ESA contribuiu substancialmente para análise e interpretação dos dados, redação do artigo, revisão crítica e aprovação da versão final do manuscrito. TMM contribuiu substancialmente para aquisição, análise e interpretação dos dados, redação do artigo e aprovação da versão final do manuscrito. MANM contribuiu substancialmente para aquisição, análise e interpretação dos dados, redação do artigo e aprovação da versão final do manuscrito. MCB contribuiu substancialmente para análise e interpretação dos dados, redação do artigo e aprovação da versão final do manuscrito.

\section{REFERÊNCIAS}

1. White KR, Forsman I, Eichwald J, Munoz K. The evolution of early hearing detection and intervention programs in the United States. Semin Perinatol. 2010 Apr;34(2):170-179.

2. Barreira-Nielsen C, Futuro Neto, HA, Gattaz G. Processo de implantação de Programa de Saúde Auditiva em duas maternidades públicas. Rev Soc Bras Fonoaudiol. 2007 Jun;12(2):99-105.

3. Bevilacqua MC, Alvarenga KF, Costa OA, Moret ALM. The universal newborn hearing screening in Brazil: from identification to intervention. Int J Pediatr Otorhinolaryngol. 2010 May;74(5):510-5.

4. Alvarenga KF, Melo TM, Lopes AC, Bevilacqua MC, Moret ALM. Participação das famílias em Programas de Saúde Auditiva: um estudo descritivo. Rev Soc Bras Fonoaudiol. 2011 Jan-Mar;16(1):49-53.

5. Korres SG, Balatsouras DG, Gkoritsa E, Eliopoulos P, Rallis E, Ferekidis E. Success rate of newborn and follow-up screening of hearing using otoacoustic emissions. Int J Pediatr Otorhinolaryngol. 2006 Jun;70(6):1039-43.

6. Mukari SZ, Tan KY, Abdullah A. A pilot project on hospital-based universal newborn hearing screening: lessons learned. Int J Pediatr Otorhinolaryngol. 2006 May;70(5):843-51.

7. Hatzopoulos S, Qirjazi B, Martini A. Neonatal hearing screening in Albania: results from an ongoing universal screening program. Int $\mathrm{J}$ Audiol. 2007 Apr;46(4):176-82.

8. Datzell LE, MacDonald MS. Follow up. In: Spivak LG (ed). Universal newborn hearing screening. New York: Thieme; 1998. p. 187-224.

9. Joint Committee of Infant Hearing, (2007). Year 2007 position statement: principles and guidelines for early hearing detection and intervention programs. Pediatrics. 2007 Oct;120(4):898-921.

10. Pryor SP, Madeo AC, Reynolds JC, Sarlis NJ, Arnos KS, Nance WE et al. SLC26A4/PDS genotype-phenotype correlation in hearing loss with enlargement of the vestibular aqueduct (EVA): evidence that Pendred syndrome and non-syndromic EVA are distinct clinical and genetic entities. J Med Genet. 2005 Feb;42(2):159-65.

11. Nance WE, Lim BG, Dodson KM. Importance of congenital cytomegalovirus infections as a cause for pre-lingual hearing loss. J Clin Virol. 2006 Feb; 35(2):221-5

12. Vohr B, McDermott C, Hess MC, Dorros C. The journey from newborn hearing screening to EHDI system: a historical perspective. In: NHS 2004 Book of Abstracts, Como, Italy, 2004. p. 2-8.

13. Assis AMO, Santos SMC, Freitas MCS, Santos JM, Silva MCM. O Programa Saúde da Família: contribuições para uma reflexão sobre a inserção do nutricionista na equipe multidisciplinar. Rev Nutr. 2002 Set;15(3):255-66.

14. Alvarenga KF, Bevilacqua MC, Martinez MANS, Melo TM, Blasca WQ, Taga MFL. Proposta para capacitação de agentes comunitários de saúde em saúde auditiva. Pro Fono. 2008 Jul-Set;20(3):171-6.

15. Melo TM, Alvarenga KF, Blasca WQ, Taga MFL. Capacitação de agentes comunitários de saúde em saúde auditiva: efetividade da videoconferência. Pro Fono. 2010 Apr-Jun;22(2):139-44.

16. World Health Organization. Report of the International Workshop on Primary Ear and Hearing Care, Cape Town, South Africa, 12-14 March, 1998. Geneva: World Health Organization; 2000.

17. World Health Organization. Primary ear and hearing care training resource. 4v. Geneva: Switzerland; 2006.

18. American Academy of Pediatrics, Council on Children with Disabilities, 
Section on Developmental Behavioral Pediatrics, Bright Futures Steering Committee and Medical Home Initiatives for Children with Special Needs Project Advisory Committee. Identifying infants and young children with developmental disorders in the medical home: an algorithm for developmental surveillance and screening. Pediatrics. 2006 July;118(1):405-20.

19. Ling D. Foundations of Spoken Language for Hearing-Impaired Children. Washington: Alex Graham Bell, Association for the Deaf; 1989.

20. Azevedo MF, Vilanova LCP, Vieira RM. Desenvolvimento auditivo de crianças normais e de alto risco. São Paulo: Plexus; 1995.

21. Newton VE, Liu X, Ke X, Xu L, Bamford JM. Evaluation of the use of a questionnaire to detect hearing loss in babies in China. Int J Pediatr Otorhinolaryngol. 1999 May 5;48(2):125-9.

22. Newton VE, Macharia I, Mugwe P, Ototo B, Kan SW. Evaluation of the use of a questionnaire to detect hearing loss in Kenyan pre-school children. Int J Pediatr Otorhinolaryngol. 2001 Mar;57(3):229-34.

23. Olusanya BO. Early detection of hearing impairments in a developing country: what options? Audiology. 2001 May-Jun;40(3):141-7.

24. Bu X, Li X, Driscoll C. The Chinese hearing questionnaire for school children. J Am Acad Audiol. 2005 Oct;16(9):687-97.
25. Stewart MG, Ohlms LA, Friedman EM, Sulek M, Duncan NO, Fernandez $\mathrm{AD}$ et al. Is parental perception an accurate predictor of childhood hearing loss? A prospective study. Otolaryngol Head Neck Surg. 1999 Mar;120(3):340-4.

26. Gomes M, Lichtig I. Evaluation of the use of a questionnaire by nonspecialists to detect hearing loss in preschool Brazilian children. Int J Rehabil Res. 2005 Jun;28(2):171-4.

27. American Academy of Family Physicians; American Academy of Otolaryngology-Head and Neck Surgery; American Academy of Pediatrics Subcommittee on Otitis Media With Effusion. Otitis media with effusion. Pediatrics. 2004 May;113(5):1412-29.

28. Northern JL, Downs MP. Hearing in children. 4th ed. Baltimore: Williams and Wilkins;1991.

29. Pagnossim DF, Oliveira TMT. Comparação das informações que os pais de diferentes classes sociais têm sobre audição. Pro fono. 1998 Mar;10(1):8-16.

30. Yoshinaga-Itano C. Levels of evidence: universal newborn hearing screening (UNHS) and early hearing detection and intervention systems (EHDI). J Commun Disord. 2004 Sep-Oct;37(5):451-65.

Anexo 1. Questionário de acompanhamento da função auditiva e de linguagem

Nome:

Área:

Família:
Data de nascimento:

Microárea:

\begin{tabular}{|c|c|}
\hline $1^{\circ}$ dia a $30 / 31$ dias de vida & $1^{\circ}$ mês \\
\hline $\begin{array}{l}\text { 1. Seu filho ouve bem? ( ) sim ( ) não } \\
\text { 2. Seu filho assusta com sons fortes? ( ) sim ( ) não }\end{array}$ & $\begin{array}{l}\text { 1. Seu filho ouve bem? ( ) sim ( ) não } \\
\text { 2. Seu filho assusta com sons fortes? ( ) sim ( ) não }\end{array}$ \\
\hline $2^{\circ}$ mês & $3^{\circ}$ mês \\
\hline $\begin{array}{l}\text { 1. Seu filho ouve bem? ( ) sim ( ) não } \\
\text { 2. Seu filho presta atenção ao som? ( ) sim ( ) não } \\
\text { 3. Seu filho reconhece a sua voz? ( ) sim ( ) não }\end{array}$ & $\begin{array}{l}\text { 1. Seu filho ouve bem? ( ) sim ( ) não } \\
\text { 2. Seu filho acalma com sua voz, com música de ninar? ( ) sim ( ) não } \\
\text { 3. Seu filho fala vogais como a, u? ( ) sim ( ) não }\end{array}$ \\
\hline $4^{\circ}$ mês & $5^{\circ}$ mês \\
\hline $\begin{array}{l}\text { 1. Seu filho ouve bem? ( ) sim ( ) não } \\
\text { 2. Seu filho tenta virar a cabeça procurando o som? ( ) sim ( ) não } \\
\text { 3. Seu filho fala mais sons que no mês anterior, por exemplo, como } \\
\text { fff, oooo? ( ) sim ( ) não }\end{array}$ & $\begin{array}{l}\text { 1. Seu filho ouve bem? ( ) sim ( ) não } \\
\text { 2. Seu filho procura os sons? ( ) sim ( ) não } \\
\text { 3. Fala vários sons como se quisesse conversar? ( ) sim ( ) não }\end{array}$ \\
\hline $6^{\circ}$ mês & $7^{\circ}$ mês \\
\hline $\begin{array}{l}\text { 1. Seu filho ouve bem? ( ) sim ( ) não } \\
\text { 2. Seu filho olha quando você chama? ( ) sim ( ) não } \\
\text { 3. Fala bababa, mamama querendo conversar? ( ) sim ( ) não }\end{array}$ & $\begin{array}{l}\text { 1. Seu filho ouve bem? ( ) sim ( ) não } \\
\text { 2. Seu filho reconhece alguns nomes da família? ( ) sim ( ) não } \\
\text { 3. Seu filho fala várias sílabas diferentes? Por exemplo: dada, papa. } \\
\text { ( ) } \operatorname{sim} \text { ( ) não }\end{array}$ \\
\hline $8^{\circ}$ mês & $9^{\circ}$ mês \\
\hline $\begin{array}{l}\text { 1. Seu filho ouve bem? ( ) sim ( ) não } \\
\text { 2. Vira rapidamente quando chamam? ( ) sim ( ) não } \\
\text { 3. Gosta de brincar com brinquedos que fazem barulhos? } \\
\text { ( ) } \operatorname{sim} \text { ( ) não }\end{array}$ & $\begin{array}{l}\text { 1. Seu filho ouve bem? ( ) sim ( ) não } \\
\text { 2. Compreende quando falam "não"? ( ) sim ( ) não }\end{array}$ \\
\hline $10^{\circ}$ mês & $11^{\circ}$ mês \\
\hline $\begin{array}{l}\text { 1. Seu filho ouve bem? ( ) sim ( ) não } \\
\text { 2. Tenta imitar os sons que fazem para ele? ( ) sim ( ) não }\end{array}$ & $\begin{array}{l}\text { 1. Seu filho ouve bem? ( ) sim ( ) não } \\
\text { 2. Dá tchau quando ouve alguém falar tchau? ( ) sim ( ) não } \\
\text { 3. Fala enrolado como se estivesse conversando? ( ) sim ( ) não }\end{array}$ \\
\hline $12^{\circ}$ mês & \\
\hline $\begin{array}{l}\text { 1. Seu filho ouve bem? ( ) sim ( ) não } \\
\text { 2. Fala as primeiras palavras? ( ) sim ( ) não } \\
\begin{array}{lll}\text { 3. Entende ordens como "cadê" e "dá"? } & \text { ( ) sim ( ) não }\end{array}\end{array}$ & \\
\hline
\end{tabular}

